\title{
Contractility of the rat cauda epididymidis and vas deferens during seminal emission
}

\author{
J. Hib, R. Ponzio and O. Vilar \\ Centro de Investigaciones en Reproducción, Facultad de Medicina, \\ Paraguay 2155-Piso 10,1121 Buenos Aires, Argentina
}

\begin{abstract}
Summary. The contractility of the cauda epididymidis and vas deferens and the movement of stained paraffin oil droplets deposited in the vas lumen were, respectively, registered and followed visually in rats electrically induced to ejaculate. Each electrical stimulation produced an abrupt contraction in the cauda epididymidis and in the middle and distal portions of the vas deferens. With the first electrical stimulations the paraffin oil droplets deposited in the proximal and middle portions of the vas deferens flowed towards the cauda epididymidis. At the moment of emission only the paraffin oil drop deposited in the distal portion of the vas was expelled towards the urethra, appearing in the urethral meatus with the ejaculated solid plug.
\end{abstract}

\section{Introduction}

The process of expulsion of the seminal fluid can be divided into two phases, namely emission and ejaculation (Baumgarten, Owman \& Sjöberg, 1975). Emission consists of movement of the spermatic fluid along the vas deferens to the pelvic urethra, and its mixing with secretions from the accessory glands. Ejaculation is the passage of this pooled seminal fluid along the penile urethra. While the organs participating in ejaculation are controlled by the parasympathetic internal pudendal nerve, emission is under the direction of the hypogastric nerve (Gruber, 1933; Semans \& Langworthy, 1938). The main nerve supply to the vas deferens is of adrenergic nature and the sympathetic fibres which reach the vas via the hypogastric nerve are mainly preganglionic, ending on ganglion cells (i.e. short adrenergic neurones) close to the ductus (Falck, Owman \& Sjöstrand, 1965; Owman \& Sjöstrand, 1965).

The problem of how the cauda epididymidis and the deferent duct contract to propel the spermatozoa towards the urethra during emission is unresolved. Several explanations based mainly on experimental findings have been proposed (see Batra, 1974, for summary). Most authors are in accord that spermatozoa coming from the testis and caput epididymidis move forward to the cauda epididymidis and proximal portions of the vas deferens, whence they will be expelled towards the urethra at the moment of emission (Risley \& Skrepetos, 1964; El-Badawi \& Schenk, 1967; Baumgarten, Holstein \& Rosengren, 1971). Spermatozoa may remain in the genital tract for considerable periods of time before being discharged at ejaculation. Nevertheless, in the absence of ejaculations the spermatozoa are permanently replaced by new ones coming from the testis, the old spermatozoa being phagocytosed by the duct mucosa cells, or moved towards the urethra by spontaneous contractions of the duct muscle cells (Montagna, 1955; Fernández-Collazo, Videla \& Pereyra, 1971; Hib, 1976). According to Baumgarten et al. (1971) at the moment of emission the spermatozoa are expelled from the cauda epididymidis and vas 
deferens by short, powerful and peristaltic contractions of the muscular walls of those ducts. However, in-vitro experiments indicated that contractions start at the urethral end of the vas deferens and spread in an epididymal direction, suggesting a sphincter at the epididymis-vas deferens junction and a consequent rise in pressure within the vas deferens that produces a violent flow of the intraluminal fluid towards the urethra (Vanwelkenhuyzen, 1966). Based on histological observations, Pabst (1969) agreed with the suggestions of Vanwelkenhuyzen (1966) but suggested that a strong contraction in the cauda epididymidis is produced to prevent the back flow of intraluminal fluid from the vas deferens.

To throw more light on this subject we have studied the contractility of the vas deferens and cauda epididymidis as well as the sperm flow after ejaculations induced by means of electrical stimulation.

\section{Materials and Methods}

Male Wistar rats, approximately 8 months old and weighing 320-360 g, were used. Each rat was anaesthetized with ethyl carbamate (urethane USP; $125 \mathrm{mg} / 100 \mathrm{~g}$ body wt) and the genital apparatus was exposed through an abdominal and scrotal incision.

In one group of 30 animals the contractility of the cauda epididymidis, the middle portion of the vas deferens and the vas portion adjacent to the urethra was registered. For this, a saline $(0.15 \mathrm{M}-\mathrm{NaCl}$ )-filled catheter (PE 10 polyethylene tubing (Intramedic), i.d. $0.28 \mathrm{~mm}$, o.d. 0.61 $\mathrm{mm}$ ) was inserted into the lumen of the ductus deferens through a small transverse incision made in the proximal or distal end of the vas and directed towards the urethra or the epididymis respectively, to reach to one of the 3 regions being studied. The lumen of the ductus was not distended by the cannula and the incisions were performed with care to preserve the vasal vessels, which accompany the principal nerves along the vas deferens. Epididymal and vasal activity were measured with a Sanborn pressure transducer $(267 \mathrm{BC})$ connected to a Hewlett-Packard preamplifier (model 350-1100), and the pressure changes were recorded on a Hewlett-Packard recorder. The amplitude of contractions (in $\mathrm{cm} \mathrm{H}_{2} \mathrm{O}$ ) was evaluated after induced ejaculations. The animals ejaculated in response to electrical stimulations administered by a single lubricated bipolar electrode inserted into the rectum, as described by Scott \& Dziuk (1959). To produce ejaculations it was necessary to apply to each rat between 3 and 16 electrical shocks, one every 5 sec, each shock lasting $3 \mathrm{sec}$.

In a second group of 15 animals the movement of the spermatozoa in the lumen of the deferent ducts was studied. With a 30-gauge surgical needle drops of liquid paraffin oil stained with Sudan black were injected into the epididymal end, into the middle portion and into the distal part of each vas deferens (Text-fig. 1a). During the ejaculations, induced as above, the transport of the tracer fluid was easily noted through the translucent wall of the vas.

\section{Results}

\section{Contractility experiments}

Each electrical stimulation was followed by an abrupt contraction at the level of the catheter tip wherever it was located. Successive stimulations produced similar responses registered by the pressure transducer. In the cauda epididymidis the average amplitude of contractions was 30.5 $\mathrm{cm} \mathrm{H}_{2} \mathrm{O}$ (range: $24-37.5$ ), in the middle portion of the vas deferens it was $110 \mathrm{~cm} \mathrm{H}_{2} \mathrm{O}$ (range: 99.5-116), and in the region of the vas adjacent to the urethra it was $85.5 \mathrm{~cm} \mathrm{H}_{2} \mathrm{O}$ (range: $72 \cdot 5-89 \cdot 5)$. 


\section{Sperm transport experiments}

Ejaculations were preceded by abrupt strong contractions of both vasa deferentia which evacuated the luminal contents, clearly visible through the translucent wall. With the first electrical stimulations the paraffin oil drop deposited in the lumen of the epididymal end of the vas deferens was ejected into the cauda epididymidis, and the drop deposited in the middle portion of the vas was moved to the proximal portion (Text-fig. 1b). At the time of emission the stained drop deposited in the distal portion of the vas was expelled towards the urethra, and appeared in the urethral meatus with the ejaculated solid plug. Approximately $90 \mathrm{sec}$ afterwards, as the muscle cells relaxed and the vas deferens diameter returned to normal, the remaining stained paraffin oil droplets returned to the place of injection into the vas (Text-fig. 1c).

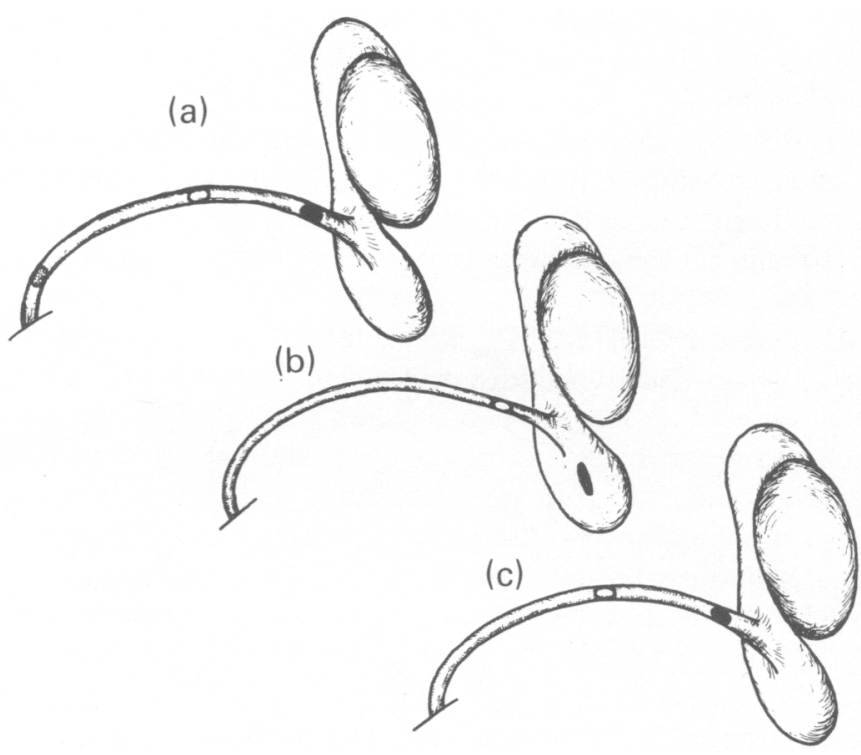

Text-fig. 1. Position of paraffin oil droplets in the rat vas deferens. (a) Before electroejaculation the droplets were located in the proximal, middle and distal portions of the vas. (b) During electroejaculation the drop located in the distal portion of the vas is expelled towards the urethra.

(c) After electroejaculation the remaining droplets returned to the place of injection in the vas.

\section{Discussion}

Our results indicate that the spermatozoa deposited in the cauda epididymidis and the proximal portions of the vas deferens in the rat do not participate in the semen composition at the moment of ejaculation. Therefore, the distal portion of the vas deferens would be the organ acting as the last reservoir for the spermatozoa before the emission. Before the ejection of these spermatozoa towards the urethra, a strong contraction of the middle and proximal portions of the vas transports the luminal contents of these portions to the cauda epididymidis, which should expand accordingly. This phenomenon could be the contractile wave from the distal to the proximal end of the vas deferens described by Vanwelkenhuyzen (1966). The entrance of spermatozoa into the cauda epididymidis during the emission was not described by Vanwelkenhuyzen (1966) or Pabst (1969). We found no evidence of the sphincter suggested by Vanwelkenhuyzen (1966), and 
Baumgarten et al. (1971) have not found any evidence of muscular structures which could serve as a sphincter at the vas-epididymal junction. Pabst (1969) suggested that the reflux of the vas intraluminal content into the cauda epididymidis at the moment of emission would be prevented by a simultaneous strong contraction in the cauda. Although our contractility recordings of the cauda epididymidis during electroejaculation support Pabst's hypothesis, we suspect that the presence of the catheter in the lumen of the vas may have distorted the hydrodynamic conditions in the cauda and, consequently, the contractile responses may have been changed. On the other hand, experimental evidence indicates that both sympathetic and parasympathetic nerves only produce contractions in the cauda epididymidis (Hib, 1976; Hib, Ponzio \& Vilar, 1979). Therefore, the distention of the cauda during the entry of the vas luminal content does not seem to be under the influence of any innervation.

The last phase of the emission depends on the abrupt contractions of the distal part of the vas deferens, and because the proximal and middle portions have already contracted, the luminal content of this distal portion is inevitably expelled towards the urethra, where the secretions of the accessory glands mix with the vas fluid. Finally, due to the reappearance of weak contractions in the cauda epididymidis, the contents that had flowed back then flowed forward again, preparing the system for the next ejaculation. Information on the storage capacity of the vas deferens is scarce, but the storage of spermatozoa in the distal portion must be important, at least in men. In vasectomized men 6-10 ejaculations are necessary before the ejaculate becomes free of spermatozoa (Freund \& Davis, 1969).

This work was supported by SECYT, Argentina. J. Hib and O. Vilar are investigators of the Consejo Nacional de Investigaciones Científicas y Técnicas de la República Argentina.

\section{References}

Batra, S.K. (1974) Sperm transport through vas deferens: review of hypotheses and suggestions for a quantita tive model. Fert. Steril. 25, 186-202.

Baumgarten, H.G., Holstein, A.F. \& Rosengren, E. (1971) Arrangement, ultrastructure, and adrenergic innervation of smooth musculature of the ductuli efferentes, Ductus epididymidis and ductus deferens of man. Z. Zellforsch. mikrosk. Anat. 120, 37-79.

Baumgarten, H.G., Owman, C. \& Sjöberg, N.O. (1975) Neural mechanisms in male fertility. In Control of Male Fertility, pp. 26-40. Eds J. J. Sciarra, C. Markland \& J. J. Speidel. Harper \& Row, New York.

El-Badawi, A. \& Schenk, E.A. (1967) The distribution of cholinergic and adrenergic nerves in the mammalian epididymis. Am. J. Anat. 121, 1-14.

Falck, B., Owman, C. \& Sjöstrand, N.O. (1965) Peripherally located adrenergic neurons innervating the vas deferens and the seminal vesicle of the guinea pig. Experientia 21, 98-100.

Fernández-Collazo, E., Videla, E. \& Pereyra, J.C. (1971) Elimination of spermatozoa in the urine of isolated male rats. J. Reprod. Fert. 27, 145-147.

Freund, M. \& Davis, J.E. (1969) Disappearance rate of spermatozoa from ejaculate following vasectomy. Fert. Steril. 20, 163-170.

Gruber, C.M. (1933) The autonomic innervation of the genito-urinary tract. Physiol. Rev. 13, 497-609.

Hib, J. (1976) Effects of autonomic drugs on epididymal contractions. Fert. Steril. 27, 951-956.
Hib, J., Ponzio, R.O. \& Vilar, O. (1979) Contractile behaviour of rat epididymis after sympathectomy produced by the administration of guanethidine. Andrologia 11, 461-465.

Montagna, W. (1955) Some cytochemical observations on human testes and epididymides. Ann. N.Y. Acad. Sci. 55, 629-642.

Owman, C. \& Sjöstrand, N.O. (1965) Short adrenergic neurons and catecholamine-containing cells in vas deferens and accessory male genital glands of different mammals. Z. Zellforsch. mikrosk. Anat. 66, 300-320.

Pabst, R. (1969) Untersuchungen über bau und funktion des menschlichen samenleiters. Z. Anat. Entwickl.Gesch. 129, 154-176.

Risley, P.L. \& Skrepetos, C.N. (1964) Histochemical distribution of cholinesterases in the testes and vas deferens of the rat. Anat. Rec. 148, 231-239.

Scott, J.V. \& Dziuk, P.J. (1959) Evaluation of the electro-ejaculation technique and spermatozoa thus obtained from rats, mice and guinea pigs. Anat. Rec. 133, 655-664.

Semans, J.H. \& Langworthy, O.R. (1938) Observations on the neurophysiology of sexual function in the male cat. $J$. Urol. 40, 836-846.

Vanwelkenhuyzen, P. (1966) La motilité du canal déférent. Acta urol. Belg. 34, 385-466.

Received 7 September 1981 